University of Wollongong

Research Online

Australian Institute for Innovative Materials -

Papers

Australian Institute for Innovative Materials

$1-1-2010$

\title{
Preparation of thiol-terminated monolayers on silicon(100) surfaces using thioacetyl-protected alkynethiol
}

\author{
Cheuk Chi Albert Ng \\ University of New South Wales \\ Guillaume Le Saux \\ University of New South Wales \\ Muthukumar Chockalingam \\ University of New South Wales \\ Simone Ciampi \\ University of New South Wales, sciampi@uow.edu.au \\ Jason Brian Harper \\ University of New South Wales
}

See next page for additional authors

Follow this and additional works at: https://ro.uow.edu.au/aiimpapers

Part of the Engineering Commons, and the Physical Sciences and Mathematics Commons

Research Online is the open access institutional repository for the University of Wollongong. For further information contact the UOW Library: research-pubs@uow.edu.au 


\title{
Preparation of thiol-terminated monolayers on silicon(100) surfaces using thioacetyl-protected alkynethiol
}

\author{
Abstract \\ The attachment of acetyl-protected alkynethiol groups onto silicon(100) surfaces was achieved using a \\ hydrosilylation methodology. Subsequent deprotection of the thiols using either hydrochloric acid or \\ ammonia solution was investigated using X-ray photoelectron spectroscopy (XPS), and compared with \\ similar reaction in solution. It was found that ammonia solution was more efficient than hydrochloric acid \\ for the deprotection step. However, the deprotection was much less efficient on the surface than in \\ solution. 2010 IEEE.

\section{Keywords} \\ 100 , surfaces, thioacetyl, protected, alkynethiol, preparation, monolayers, thiol, terminated, silicon \\ Disciplines \\ Engineering | Physical Sciences and Mathematics

\section{Publication Details} \\ Ng, C. Chi Albert., Le Saux, G., Chockalingam, M., Ciampi, S., Harper, J. B. \& Gooding, J. Justin. (2010). \\ Preparation of thiol-terminated monolayers on silicon(100) surfaces using thioacetyl-protected \\ alkynethiol. ICONN 2010 - Proceedings of the 2010 International Conference on Nanoscience and \\ Nanotechnology (pp. 244-247). United States: Institute of Electrical and Electronics Engineers.

\section{Authors} \\ Cheuk Chi Albert Ng, Guillaume Le Saux, Muthukumar Chockalingam, Simone Ciampi, Jason Brian Harper, \\ and J Justin Gooding
}




\title{
Preparation of thiol-terminated monolayers on silicon(100) surfaces using thioacetyl-protected alkynethiol
}

\author{
Cheuk Chi Albert Ng, Guillaume Le Saux, Muthukumar Chockalingam, Simone Ciampi, Jason B. Harper and \\ J. Justin Gooding* \\ *Corresponding author. School of Chemistry, University of New South Wales, Sydney NSW 2052, Australia
}

\begin{abstract}
The attachment of acetyl-protected alkynethiol groups onto silicon(100) surfaces was achieved using a hydrosilylation methodology. Subsequent deprotection of the thiols using either hydrochloric acid or ammonia solution was investigated using $\mathrm{X}$ ray photoelectron spectroscopy (XPS), and compared with similar reaction in solution. It was found that ammonia solution was more efficient than hydrochloric acid for the deprotection step. However, the deprotection was much less efficient on the surface than in solution.
\end{abstract}

Keywords- silicon; hydrosilylation; thiol; protection group; self-assembled monolayer

\section{INTRODUCTION}

Surfaces presenting thiol functional groups have applications in molecular electronics, $[1,2]$ biochemistry, $[3,4]$ and gold nanoparticle attachment.[5] For many of these applications, there is a need to present the functional groups on the surface in an ordered and controllable way. Self-assembly monolayers (SAMs) are an excellent platform for this purpose.

In 1995, Linford et al. showed that 1-alkenes and 1-alkynes could react with silicon(111) surfaces by a thermal hydrosilylation reaction to form stable and well-ordered SAMs where the bond at the silicon surfaces was via a Si-C linkage.[6] This kind of SAM is more robust and chemically stable than either traditional alkanethiol or silane SAMs.[6]

Sieval et al. showed that the hydrosilylation reaction can be performed on silicon(100) surfaces as well to produce good quality SAMs.[7] However, if the alkene or alkyne contains functional groups that could react with the hydrogenterminated silicon surface, inhomogeneous monolayers would be formed, and the desired functional group would be lost.[7]

Since thiols can react with the hydrogen-terminated silicon surfaces, the thiol group needed to be protected.[1,5] The use of the trifluoroacetyl protection group for this purpose has been reported previously. [1,5] The advantages of this protecting group are that it is very easy to remove and does not affect the packing of the SAM by steric hindrance [1]. However, the trifluoroacetyl-protected alkynes and alkenes are not commercially available. Also, they are prone to hydrolysis.
To strike a balance between stability of the protected precursor and ease of deprotection on surface, the potential of the acetyl protection group as such a candidate would be explored in this paper.

\section{EXPERIMENTAL SECTION}

\section{Materials}

Silicon wafers (Virginia Semiconductor, Inc.) were double side polished, boron doped, $0.50 \mathrm{~mm}$ thick, resistivity 0.001 $0.005 \Omega \mathrm{cm}$. Sulfuric acid, hydrofluoric acid and hydrogen peroxide (Riedel de Hahn, Sydney, Australia) for the hydrosilylation reaction were semiconductor grade. Sodium hydroxide (analytical reagent grade), magnesium sulfate, hydrochloric acid (32\%) and ammonia (28\%) were obtained from Ajax Finechem, Sydney, Australia. Organic solvents were distilled before use. Thioacetate 1 was synthesised using the procedure reported previously.[8] Thin-layer chromatography (TLC) was performed on Merck silica gel 60F234 aluminium sheets. All other chemicals, unless stated otherwise, were used as received without further purification.

\section{Deprotection of the thioacetate 1 in solution using acid (figure 1) \\ The details of deprotection of the thioacetate 1 using hydrochloric acid were reported earlier.[1, 9] In brief, the thioacetate 1 was dissolved in deoxygenated 95\% ethanol : $32 \% \mathrm{HCl}(75: 1, \mathrm{v} / \mathrm{v})$ and heated at reflux under nitrogen atmosphere. The extent of deprotection was monitored using TLC, until no starting materials could be detected.}

Deprotection of the thioacetate 1 in solution using base (figure 1)

To a 3-neck flask under nitrogen atmosphere was loaded the thioacetate 1 ( $c a .40 \mathrm{mg})$. Either sodium hydroxide (2 M deoxygenated aqueous solution) or ammonia $(15 \mathrm{M}$, diluted 
with $5 x(v / v)$ deoxygenated $95 \%$ ethanol) was added. The reaction mixture was then stirred overnight under nitrogen at room temperature. After that, the reaction mixture was acidified to around $\mathrm{pH} 6$ with hydrochloric acid $(10 \mathrm{M})$, monitored by $\mathrm{pH}$ paper. The mixture was then diluted with water $(c a .5 \mathrm{~mL})$ and extracted with ether $(3 \times 10 \mathrm{~mL})$. The organic portion was dried $\left(\mathrm{MgSO}_{4}\right)$, and evaporated in vacuo to obtain the crude thiol 2 . The reaction yield was estimated using ${ }^{1} \mathrm{H}$ NMR (300 MHz, $\mathrm{CDCl}_{3}$ ), by comparing the intensity of the peak around $\delta 2.84\left(\mathrm{t}, 2 \mathrm{H}, J=7.1 \mathrm{~Hz}, \underline{\mathrm{CH}}_{2} \mathrm{SAc}\right.$ in 1) and the peak at around $\delta 2.48$ (q, $2 \mathrm{H}, J=7.5 \mathrm{~Hz}, \underline{\mathrm{CH}}_{2} \mathrm{SH}$ in 2).

\section{Preparation of surfaces}

The preparation of surface S2 (figure 2) was reported previously.[8,9] Surface S2 was immersed in distilled (under reduced pressure), degassed (at least 5 freeze-pump-thaw cycles) thioacetate $\mathbf{1}$ in a Schlenk flask under argon at $120^{\circ} \mathrm{C}$ overnight to afford surface $\mathbf{S 3}$. Surfaces $\mathbf{S 3}$ were then placed in either $15 \mathrm{M}$ ammonia solution or $10 \mathrm{M}$ hydrochloric acid to afford surface $\mathbf{S 4}$.

\section{XPS measurements and analysis}

XPS of the surfaces were acquired using an ESCALAB 220iXL spectrometer with a monochromatic Al K $\alpha$ source, hemisperical analyzer and multichannel detector. Binding energies were corrected with reference to Si 2p1/2 signal (99.9 $\mathrm{eV})$. Survey scans $(0-1100 \mathrm{eV})$ were carried out with $1.0 \mathrm{eV}$ step size, $100 \mathrm{~ms}$ dwell time, and pass energy $100 \mathrm{eV}$. Highresolution scans (Si 2p, S 2s, C 1s) were carried out with 0.1 eV step size, $100 \mathrm{~ms}$ dwell time, and pass energy $20 \mathrm{eV}$.

The XP spectra were analysed using the curve-fitting program XPSPEAK 4.1. The high-resolution scans were first background-subtracted using the Shirley method, then deconvoluted and fitted with peaks with mixed GaussianLorentzian functions. The error of peak-fitting in each highresolution scan region was represented by the $\chi^{2}$ value. These $\chi^{2}$ values were less than 1 , indicating accurate fits. Atomic compositions of the surfaces were calculated using the peak area, number of scans, and atomic sensitivity of the elements.[9]
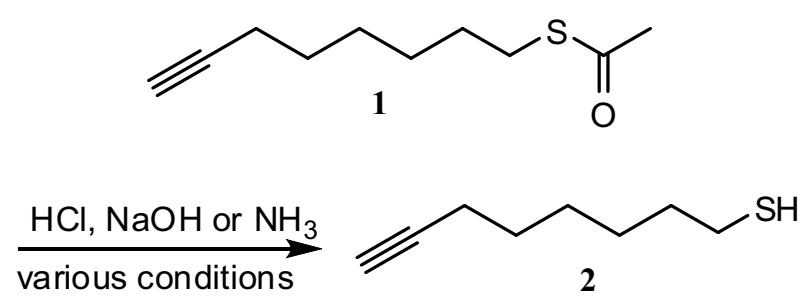

Fig. 1: deprotection of the thioacetate 4 to afford thiol 5

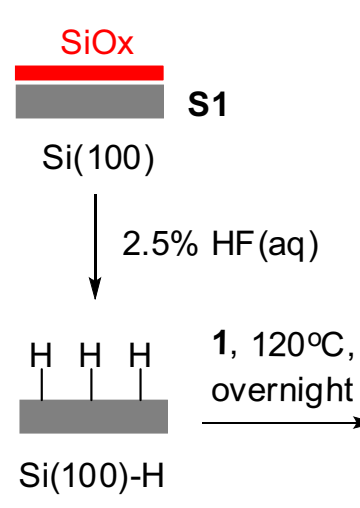

S2
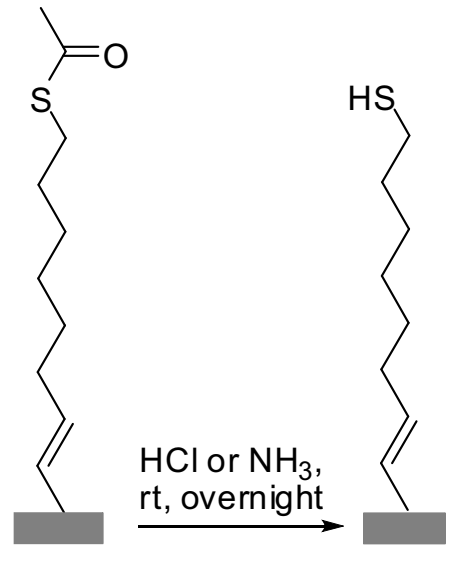

Fig. 2: Attachment of the protected thiol. 1 and subsequent reactions on Si(100) surfaces.

\section{RESULTS AND DISCUSSION}

\section{Deprotection of thioacetate 1 in solution}

The deprotection strategy needed to be established in bulk solution before applying to deprotection on surfaces. Several methods were attempted to remove the acetyl protecting group to form the thiol 2 (figure 1). Deprotection using hydrochloric acid (ca. $0.1 \mathrm{M}$ ethanoic solution) were reported previously by Ciampi et al., reacting at $60^{\circ} \mathrm{C}$ for $5 \mathrm{~h}$ and achieved $63 \%$ yield.[10] However, to achieve complete deprotection, the reaction required $48 \mathrm{~h}$.

Subsequently, a basic deprotection procedure was explored. Using sodium hydroxide, reacting at room temperature overnight achieved approximately $20 \%$ deprotection. Alternatively, using ammonia, reacting at room temperature overnight achieved $>95 \%$ deprotection. The above results show that ammonia is the most effective of the three reagents investigated for this process.

\section{Attachment of thioacetate 1 to Si(100) to give surface $\mathbf{S 3}$}

Figure $3 \mathrm{a}$ shows the $\mathrm{C} 1 \mathrm{~s}$ high resolution XP spectrum of surface S3. The spectrum can be deconvoluted into three peaks: (i) $c a .285 \mathrm{eV}$, due to carbon atoms bonded to either another carbon atom or a silicon atom [1]; (ii) $c a$. $286.5 \mathrm{eV}$, due to either carbon atoms bonded to a sulfur atom [1] or oxidized carbon contamination [11]; (iii) ca. $288.5 \mathrm{eV}$, due to carbonyl carbons bonded to a sulfur atom [1].

Figure 4 shows the S 2s high resolution XP spectrum of surface S3, with a single peak at $c a$. $228.5 \mathrm{eV}$. It is about 0.5 $\mathrm{eV}$ lower than the sulfur atom bonded to trifluoroacetyl protecting group $[1,8]$. This may due to a lower electronwithdrawing ability of the acetyl group, compared with the trifluoroacetyl group, bonded to the sulfur atom. 
The present of all three $\mathrm{C} 1 \mathrm{~s}$ and the $\mathrm{S} 2 \mathrm{~s}$ peaks showed the attachment of thioacetate 1 onto the surface was successful.

\section{Deprotection of thioacetate moiety on surface $\mathbf{S 3}$}

After showing that both hydrochloric acid and ammonia could achieve nearly complete deprotection in solution, both deprotection strategies were investigated for deprotection of surface bounds molecules, surface $\mathbf{S 3}$. Figure $3 \mathrm{~b}$ shows the XP spectra of surface $\mathbf{S 4}$, formed by immersing $\mathbf{S 3}$ in hydrochloric acid (10 $\mathrm{M}$ aqueous solution) overnight, while figure $3 \mathrm{c}$ showed the XP spectra of another surface S4, formed by immersing $\mathbf{S 3}$ in ammonia (15 M aqueous solution) overnight. Comparing to figure $3 \mathrm{a}$, the signal corresponding to the carbonyl carbon ( $c$ a. $288.5 \mathrm{eV}$ ), did not decrease significantly after hydrochloric acid treatment, but did decrease significantly after ammonia treatment. In contrast, the S 2s peak intensity was almost unchanged after undergoing either deprotection treatment. Quantitative analysis showed that the ratio of the carbonyl carbon to sulfur (calculated using the area of the carbonyl carbon peak and the area of S 2 s peak, adjusted by the relative atomic sensitivity of sulfur and carbon) was 0.99:1 in S3, 0.87:1 after hydrochloric acid treatment and 0.46:1 after ammonia treatment. Therefore, the extent of deprotection was about 13\% using hydrochloric acid and about 54\% using ammonia. Similar to the reaction in solution, ammonia is more effective than hydrochloric acid for removing the acetyl group. However, these results also showed that the rate of deprotection on surface is significantly lower than that in solution.

\section{CONCLUSION}

The above results show that ammonia is more effective than hydrochloric acid for removing the acetyl protecting group. While complete deprotection of this group is relatively easy to achieve in solution, complete deprotection on surfaces is less efficient. This inefficiency becomes more noticeable when compared to the trifluoroacetyl group, where only 10 mins at room temperature in aqueous ammonia is required to achieve complete deprotection.[1,5] Therefore, to prepare thiolterminated SAMs on silicon surface, if the synthetically straightforward acetyl derivative is used, a much longer deprotection time and a higher deprotection temperatures are needed. Otherwise, the more synthetically challenging trifluoroacetyl derivative is required. (i)

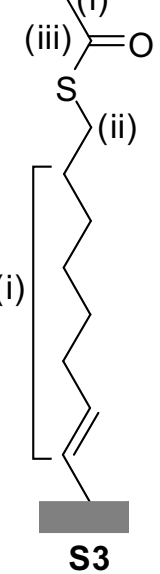

(a)

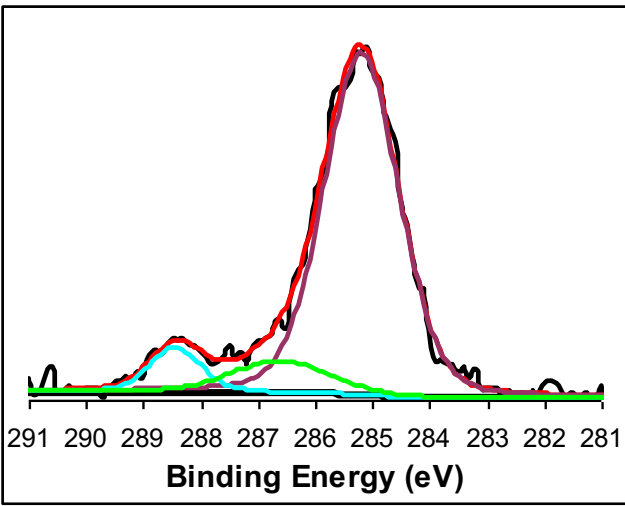

S3

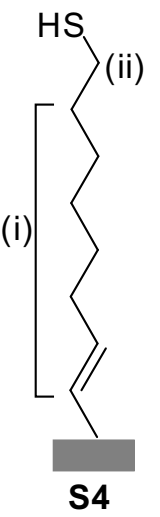

(b)

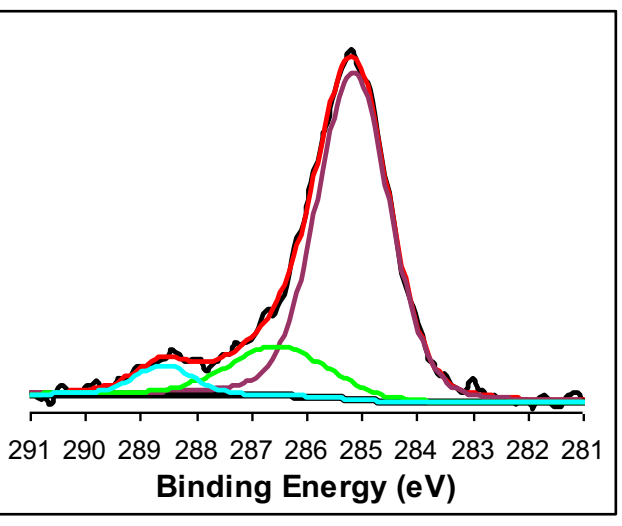

S4

(c)

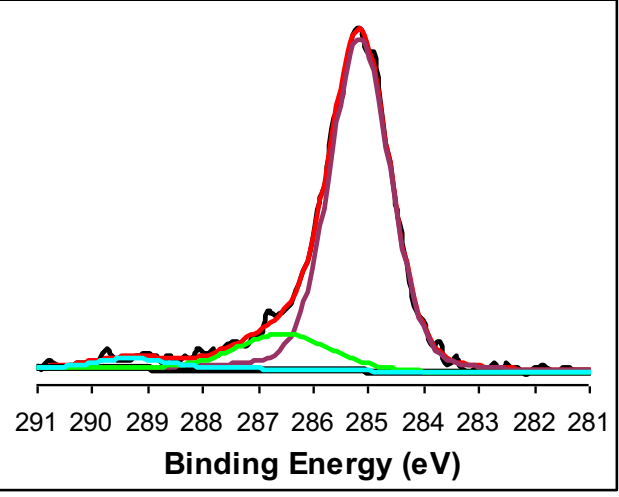

Fig. 3: C 1s high resolution XP spectra of surfaces: (a) S3; (b) $\mathbf{S 4}$ by $\mathrm{HCl}$ route; (c) $\mathbf{S} 4$ by $\mathrm{NH}_{3}$ route. Peak assignment key: (i) purple; (ii) green; (iii) blue 


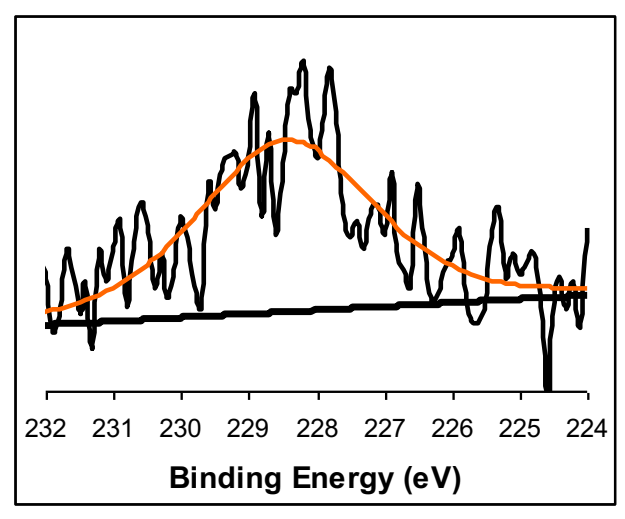

Fig. 4: S 2s high resolution XP spectrum of surface $\mathbf{S 3}$. The S 2s spectra of S4 (by $\mathrm{HCl}$ or $\mathrm{NH}_{3}$ route) are very similar.

\section{ACKNOWLEDGMENT}

This research was supported by the Australian Research Council's Discovery Projects funding scheme (project number DP0772356). A.N. was supported by an Australian Postgraduate Award from the Australian Government. We also thank Dr Bill Bin Gong for help with X-ray photoelectron spectroscopy.

\section{REFERENCES}

[1] Bocking, T.; Salomon, A.; Cahen, D.; Gooding, J. J., Langmuir, 2007, 23, 3236-3241.

[2] Salomon, A.; Böcking, T.; Gooding, J. J.; Cahen, D., Nano Lett., 2006, 6, 2873-2876

[3] Wang, Y.; Yao, X.; Wang, J.; Zhou, F., Electroanalysis, 2004, 16, 1755 1761 .

[4] Lenigk, R.; Carles, M.; Ip, N. Y.; Sucher, N. J., Langmuir, 2001, 17, 2497-2501.

[5] Le Saux, G.; Ciampi, S.; Gaus, K.; Gooding, J. J., Appl. Mater. Interfaces, 2009, 1, 2477-2483.

[6] Linford, M. R.; Fenter, P.; Eisenberger, P. M.; Chidsey, C. E. D., J. Am. Chem. Soc., 1995, 117, 3145-3155.

[7] Sieval, A. B.; Demirel, A. L.; Nissink, J. W. M.; Linford, M. R.; van der Maas, J. H.; de Jeu, W. H.; Zuilhof, H.; Sudholter, E. J. R., Langmuir, 1998, 14, 1759-1768.

[8] Ng, A.; Ciampi, S.; James, M.; Harper, J. B.; Gooding, J. J., Langmuir, 2009, 25, 13934-13941.

[9] Ciampi, S.; Bocking, T.; Kilian, K. A.; James, M.; Harper, J. B.; Gooding, J. J., Langmuir, 2007, 23, 9320-9329.

[10] Ciampi, S.; Eggers, P. K., Le Saux, G.; James, M.; Harper, J. B.; Gooding, J. J., Langmuir, 2009, 25, 2530-2539.

[11] Cerofolini, G. F.; Galati, C.; Reina, S.; Renna, L., Surf. Interface Anal., 2006, 38, 126-138 